\title{
Bilateral Six Cusped and Three Rooted Mandibular First Molars
}

\author{
${ }^{1}$ Anantharaj A, ${ }^{2}$ Praveen P, ${ }^{2}$ Karthik Venkataraghavan, ${ }^{3}$ Prathibha Rani S, ${ }^{4}$ Murali B Krishnan \\ ${ }^{1}$ P rofessor and Head, Department of Pediatric and Preventive Dentistry, DAPM RV Dental College, Bengaluru, Karnataka, India \\ ${ }^{2}$ Professor, Department of Pediatric and Preventive Dentistry, DAPM RV Dental College, Bengaluru, Karnataka, India \\ ${ }^{3}$ Senior Lecturer, Department of Pediatric and Preventive Dentistry, DAPM RV Dental College, Bengaluru, Karnataka, India \\ ${ }^{4}$ P ostgraduate Student, Department of Pediatric and Preventive Dentistry, DAPM RV Dental College, Bengaluru, Karnataka, India
}

Correspondence: Anantharaj A, Professor and Head, Department of Pediatric and Preventive Dentistry, DAPM RV Dental College Bengaluru, Karnataka, India, Phone: 09845166435, e-mail: dranantharaj@gmail.com

\section{ABSTRACT}

Teeth are vertebrate organs that arise from complex and progressive interactions between an ectoderm, the oral epithelium and an underlying mesenchyme. A significant amount of research has focused on determining the processes that initiate tooth development. It is widely accepted that there is a factor (multiple signaling molecules, including BMPs, FGFs, Shh and Wnt proteins) within the tissues of the first branchial arch that is necessary for the development of teeth. A 9-year-old reported to our department with the chief complaint of pain in the lower right back teeth region. On clinical examination, mandibular molars revealed the presence of an extra cusp on the lingual surface. Occurrence of six cusps in permanent mandibular molar is a rare phenomenon, and number of cases reported is very few.

Keywords: Teeth, Cusp, First branchial arch.

\section{INTRODUCTION}

Human teeth of both dentitions may show variations and changes in morphological structures. Such changes may be found on the crown either in the form of anomalous cusps or in an increased number of roots, which in some instances are associated with an anomalous cusp. It is generally believed that the numerous morphologic characteristics of the teeth are genetically determined. ${ }^{1,2}$ Detailed description and study of these traits could provide valuable information regarding phylogeny of man and distinctions between races and sub races. ${ }^{1,3-12}$ The study of the number of cusps is not only important in anthropology, it is also important in the study of dental occlusion, orthodontics, restorative dentistry and prosthetic dentistry.

In modern man, the number of cusps of the upper molars is frequently reduced from four to three. The formula for the number of cusps of the upper molar series is changing from 44-4 to 4-4-3 and 4-3-3. The trend is more marked in females than in males of all populations that have been studied. In most studies, mal es generally showed a lesser tendency than females to reduction in the number of cusps of the upper molars. The process of reduction occurs through the elimination of the hypocone or distolingual cusp, with a subsequent decrease in the mesiodistal crown diameter and in the occlusal area. ${ }^{13}$ Similarly, the number of cusps can increase, and this is attributed to local proliferation of enamel knot.

\section{CASE REPORT}

A 9-year-old child reported to the Department of Pediatric Dentistry, DAPM RV, Dental College, Bengaluru, with a complaint of pain in the lower left back teeth region since 15 days. On examination, there was grossly decayed lower left

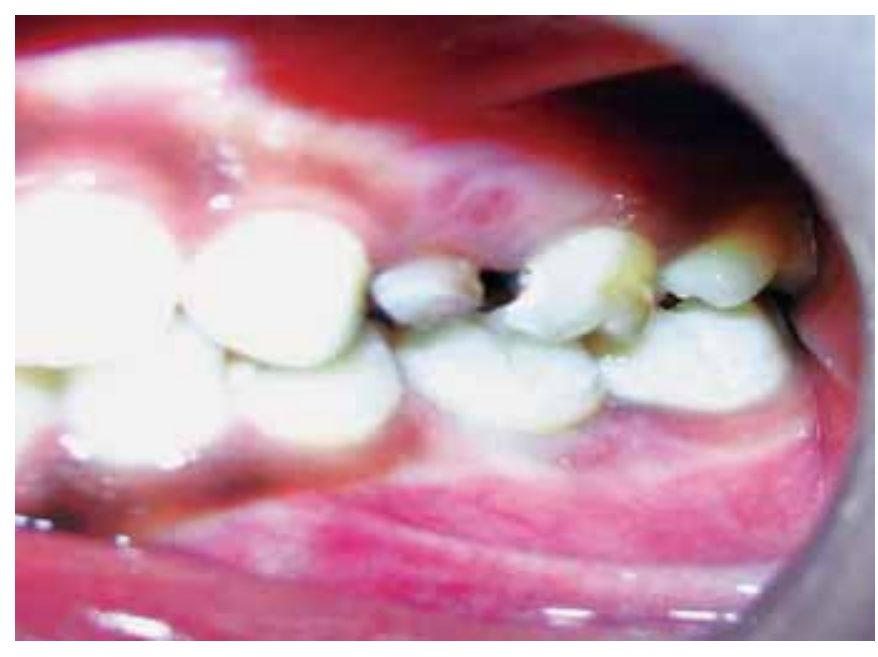

Fig. 1: Left side showing Angle's class I molar relation

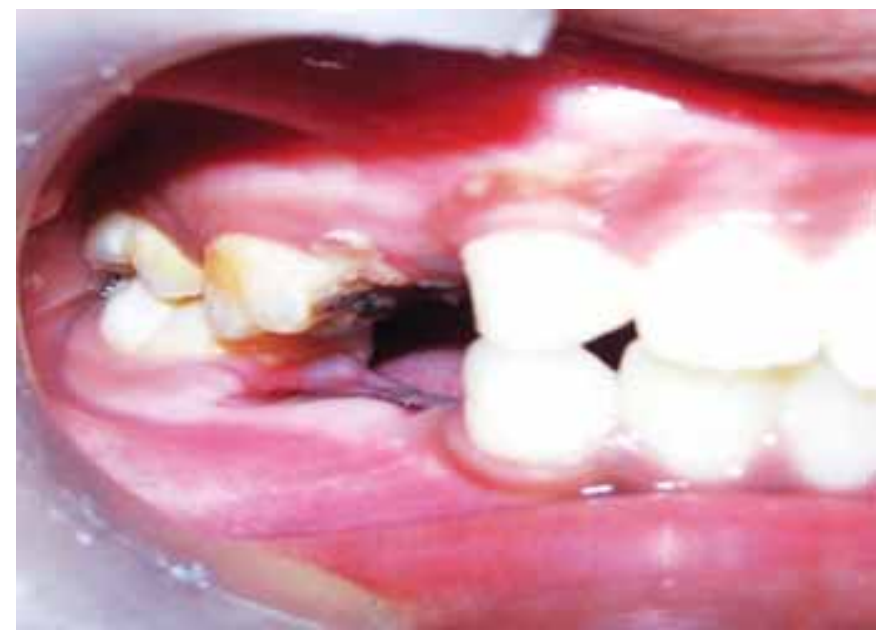

Fig. 2: Right side showing Angle's class I molar relation 


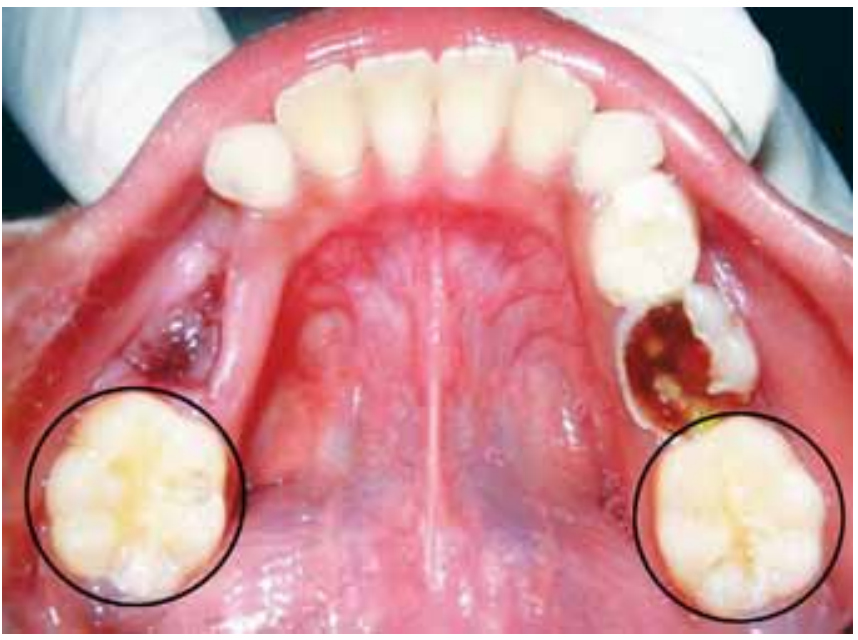

Fig. 3: Mandibular first permanent molar exhibiting six cusps

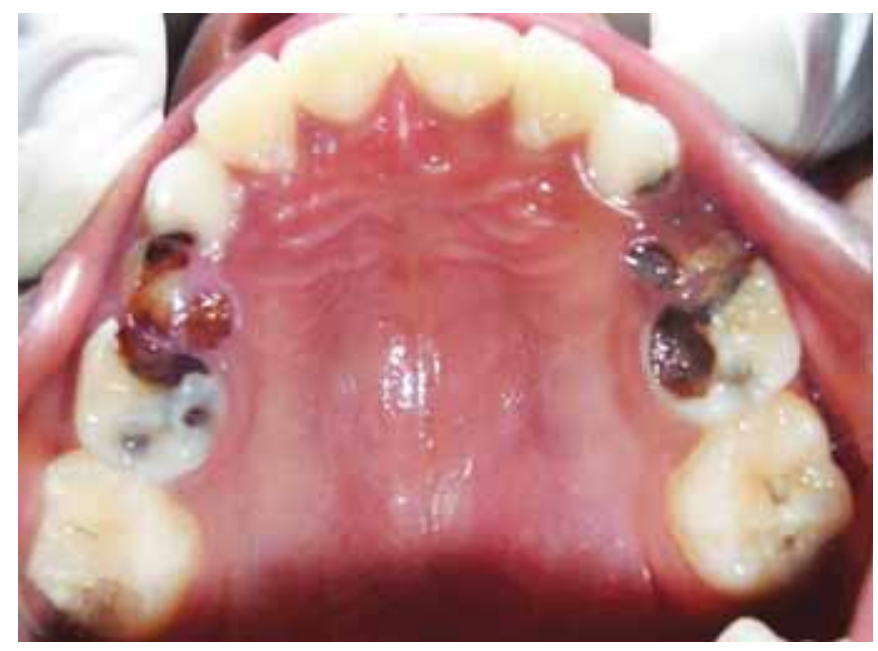

Fig. 4: Maxillary arch showing four cusped first permanent molar

second deciduous molar. M olar relation was found to be Angle's class I bilaterally (Figs 1 and 2). A nother striking finding was the presence of six cusps in permanent mandibular first molar (Fig. 3). The permanent maxillary first molar had four cusps (Fig. 4). There was no positive family history. The six cusped molar exhibited deep pit and fissures which were susceptible for dental caries, and hence pit and fissure seal ant was applied. A I IOPA of mandibular first molar revealed the presence of an extra root (Figs 5 and 6 ). The extra root was located in the buccal aspect (localized, using SLOB technique).

\section{DISCUSSION}

The patterns of the occlusal surfaces of molars are polygenically conditioned and determined by a combination of allele on two or more sites/loci, and they occur in one of the final stages of molar growth, as a result of the terminal deposition of enamel. ${ }^{1}$ Numerous studies have shown that the genes of the $X$ chromosome regulate the deposition of enamel, while genes of the $Y$-chromosome influence the division of the cell connected with the formation of the dentine-enamel bond and the deposition of enamel. ${ }^{14}$

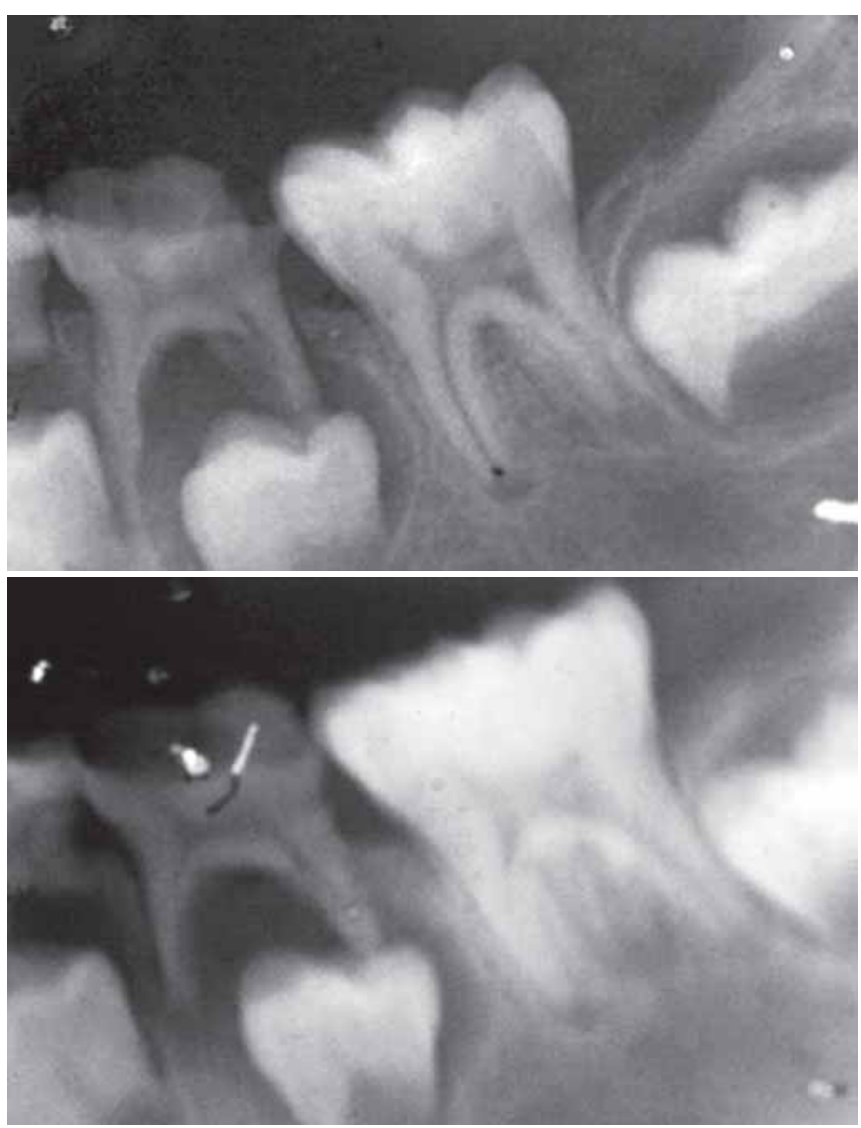

Fig. 5: IOPA of 36 region using localization technique reveals the presence of radix paramolaris
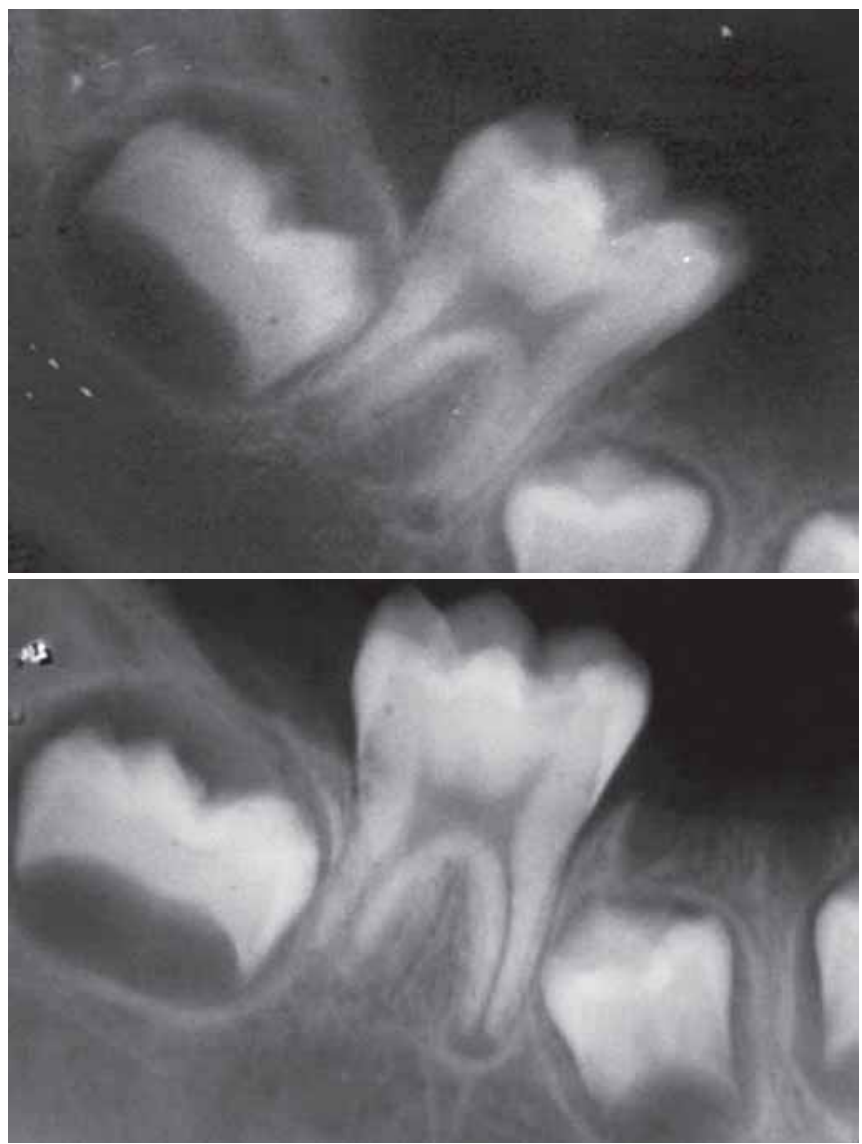

Fig. 6: IOPA of 46 region using localization technique reveals the presence of radix paramolaris 
Bilateral Six Cusped and Three Rooted Mandibular First Molars

The mandibular first molar generally has five cusps, this trait describes the presence of only three or four cusps. The four cusp variant occurs with the absence of the distobuccal cusp, the three cusp variant has missing the distolingual cusp as well. If the five common cusps are present, other variations exist in the presence of a sixth or seventh cusp at the distobuccal and distolingual surfaces. These latter two traits are rare in humans but can be more common among some of our hominid ancestors.

The fifth or distobuccal cusp is the most variable, which may be absent. If the fifth cusp is present, it is often placed lingually, out of line with the other two buccal cusps. The fifth cusp may be divided into two parts by a fissure which runs anteroposteriorly, thus the tooth may have three lingual and three buccal cusps. A total of ten cusps can be theoretically present on a lower molar tooth. There is a large amount of publications on molar morphology, numbers of molar cusps, origins, and evolution of both cusps and teeth. ${ }^{14}$ There are no standards yet available to determine whether a cusp should be recorded as one because of its inadequate size.

Suzuki and Sakai ${ }^{15}$ found no significant differences in first and second molar cusp numbers of 392 male and female Japanese.

The frequency of cusp six on first and second molars (65 and 63\% respectively) from Indian crania was found to be about three times that of A leuts ( 21 and $18 \%$ ) and Eskimos (22 and 18\%). All three groups (Indian, A leuts and Eskimos) showed five or six cusped first lower molars. No four cusped molars were found. F our-cusped second lower molars occurred most often in the A leuts (9\%), less in Indians (3\%) and least in the Eskimos (1\%). ${ }^{16}$

A ccording to the number of cusps, a tooth can be classified as follows:

1. The presence of cusps 1-4 (1-protoconid; 2-metaconid; 3-hypoconid; 4-entoconid).

2. The presence of five cusps (hypoconulid)

3. The presence of six cusps (entoconulid).

The mandibular molars are more variable than the maxillary molars in the number of different patterns and in the frequency of these patterns. The typical $Y 5$ pattern is seen in $62.1 \%$ of the total population; however $28.1 \%$ exhibit the $Y 6$ pattern, that is, the $Y$ groove shape with a sixth cusp. The frequency of +5 and +6 is relatively low and only $0.6 \%$ exhibit the four cusp pattern. No ethnic differences were found, but there is a significant $(0.005<p<0.0005$, 1f) sex effect, principally, because males exhibit the six cusp pattern more frequently. ${ }^{17}$

Like the number of root canals, the number of roots may also vary. A $n$ additional third root, first mentioned in the literature by Carabelli is called the radix entomolaris (RE). This supernumerary root is located distolingually in mandibular molars, mainly first molars. A $n$ additional root at the mesiobuccal side is called the radix paramolaris (RP). ${ }^{9}$
The prevalence of extra root in mandibular first molar is as follows. 'Radix Entomolaris' (RE) in first mandibular molar is associated with certain ethnic groups.

- In African populations a maximum frequency of $3 \%$ is found, while in Eurasian and Indian populations the frequency is less than $5 \%$

- Survey of population of M ongoloid origin indicates a high prevalence, ranges from 5 to more than $30 \%$

- Bolk reported that the occurrence of radix paramolaris is very rare and occurs less frequently than the radix entomolaris

- Curzon (1974) suggested that the 'three rooted molar' trait has a high degree of genetic penetrance as its dominance

- Its formation could be related to external factors during odontogenesis. ${ }^{10}$

\section{CONCLUSION}

The presence of increased number of cusps has been reported in permanent maxillary first molar, mandibular second, third molars and also in deciduous mandibular molars. Accessory cusps have been reported in permanent mandibular first molars in hominoid ancestors only. Clinical implication of this extra cusp in the present case is that the occlusal table of the tooth is increased with deep pit and fissures. Hence, the chances of food getting lodged are high. This in turn increases the decay activity also. So, pit and fissure sealants were placed in both the lower permanent molars, pulp therapy performed in lower left second deciduous molar, extraction of upper first deciduous molars and stainless steel crown in upper second deciduous molars. Patient was recalled for periodic check-up.

\section{REFERENCES}

1. M oorrees CFA. The Aleut dentition. Cambridge: Harvard University Press 1957.

2. L undstrom A. Tooth morphology as a basis for distinguishing monozygotic and dizygotic twins. Swed Med Res Counc 1962;34-43.

3. Hrdlicka A. Shovel-shaped teeth. Am J Phys Anthropol 1920;3:429-66.

4. Hrdlicka A. Further studies of tooth morphology. Am J Phys Anthropol 1921;4:141-76.

5. Dahl berg AA. The dentition of the A merican Indian. In: Laughlin WS (Ed). Dental anthropology. Papers on the physical anthropology of the A merican Indian. Fourth Viking Fund Summer Seminar. TheV iking Fund Inc., New Y ork 1951; 138-76.

6. Lasker GW . Observations on the teeth of Chinese born and reared in China and A merica. A m J Phys A nthropol 1945;3:129-50.

7. Lasker GW. Genetic analysis of racial traits of the teeth. Cold Spring Harbor Symposia on Quantitative Biology, XV 1951;191-203.

8. Tratman EK. A comparison of the teeth of people: IndoEuropean racial stock with the Mongoloid. Dent $\mathrm{Rec}$ 1950;70:63-88

9. M oorrees CFA. G enetic considerations in dental anthropology. Genetics and dental health. New Y ork: M c Graw Hill 1962; 101-12.

10. Carbonell VM . V ariations in the frequency of shovel-shaped incisors in different populations. In: B rothwell DR (Ed). Dental A nthropology. Symposia of the Society for the Study of Human Biology. N ew Y ork: Pergamon Press 1963;5. 
11. Palomino H, Chakraborty R, R othhammer F. D ental morphology and population diversity. Hum Biol 1977;49:61-70.

12. Greene DL. Genetics, dentition and taxomony. U niv W yom Pub 1967;33:93-168.

13. John Y K, Ling. Molar cusps in Southern Chinese. The Open Anthropology Journal 2010;3:16-19.

14. Mihajlo M aćešić. Characteristics of the occlusal surfaces of lower molars in a sample of the croatian population. A cta Stomat Croat 2003;69-73.
15. Suzuki, Sakai. Occlusal surface pattern of the low er molars and the second deciduous molar among the living Polynesians. A merican journal of physical anthropology 1973.

16. T urner II CG. The dentition of the A rctic peoples. Dissertation. Univ Wisconsin, M adison 1967.

17. Hernan Palomino. The A ymara of W estern B olivia: Occlusion, pathology, and characteristics of the dentition (III). J Dent Res 1978;57:459. 